\title{
Behavioral and Brain Sciences
}

http://journals.cambridge.org/BBS

Additional services for Behavioral and Brain Sciences:

Email alerts: $\underline{\text { Click here }}$

Subscriptions: $\underline{\text { Click here }}$

Commercial reprints: Click here

Terms of use : Click here

\section{Why homolaterality of language and hand dominance may not be the expression of a specific evolutionary link}

Bencie Woll and Jechil S. Sieratzki

Behavioral and Brain Sciences / Volume 26 / Issue 02 / April 2003, pp 241 - 241

DOI: 10.1017/S0140525X03570066, Published online: 02 October 2003

Link to this article: http://journals.cambridge.org/abstract S0140525X03570066

How to cite this article:

Bencie Woll and Jechil S. Sieratzki (2003). Why homolaterality of language and hand dominance may not be the expression of a specific evolutionary link. Behavioral and Brain Sciences,26, pp 241-241 doi:10.1017/S0140525X03570066

Request Permissions : $\underline{\text { Click here }}$ 
Commentary/Corballis: From mouth to hand: Gesture, speech, and the evolution of right-handedness

scanning tools became available; e.g., Tanaka et al. 1999). Although as Good et al. (2001) point out, there is a strong presumption throughout the neuroanatomical literature that all structural left-right asymmetries strongly indicate functional asymmetries, there are many inconsistencies in textbook accounts, including the larger frontal lobe of the nondominant hemisphere and the lack of gender differences in language performance to parallel the sex differences found in degree of structural asymmetry (Good et al. 2001; Walker 1980). The most reasonable conjecture based on the studies above would, I suggest, be the acceptance of the null hypothesis for the relationship between structural and functional left-right asymmetries in the human brain. This in itself would have little impact on Corballis's claim that functional asymmetries for spoken language lead the human population asymmetry in hand preference. Indeed, accepting that some of the volume asymmetries in human and great ape brains are unrelated in either case to functional language specializations would solve problems that Corballis otherwise has with Cantalupo and Hopkins (2001) and Pilcher et al. (2001). Kennedy et al. (1998) suggested that the major source of variance in human cortical volume is individual differences applying to individual gyri, which is relatively independent of larger-scale variation; and that, in particular, local variations in the frontal and temporal language specific regions do not correlate well with total cortical volume.

Much of the target article is speculation which may never be disconfirmed by evidence. But there are accumulating data on the (largely conserved) genetic factors that control structural asymmetries of the kind that are disturbed in situs inversus (Hamada et al. 2002; Hobert et al. 2002; Mercola \& Levin 2001) and the faint beginnings of knowledge of the genetic factors responsible for uniquely human capacities, some of which often, but not always, display left-right asymmetry (Ennard et al. 2002; though see, e.g., Meaburn et al. 2002). A detailed molecular account of the extent to which speech entails handedness may therefore be eventually attainable, but it is unlikely to correspond very closely to Corballis's narrative.

\section{Why homolaterality of language and hand dominance may not be the expression of a specific evolutionary link}

\section{Bencie Wolla and Jechil S. Sieratzkib \\ aDepartment of Language and Communication Science, City University London, London EC1V OHB, United Kingdom; ${ }^{\mathrm{b}}$ Department of Human Communication Science, University College London, London WC1, United Kingdom.b.woll@city.ac.uk sieratzki@vff.uni-frankfurt.de www.city.ac.uk/lcs}

\begin{abstract}
Although gestures have surface similarities with language, there are significant organisational and neurolinguistic differences that argue against the evolutionary connection proposed by Corballis. Dominance for language and handedness may be related to a basic specialisation of the left cerebral hemisphere for target-directed behaviour and sequential processing, with the right side specialised for holistic-environmental monitoring and spatial processing.
\end{abstract}

Gesture and language are separated by fundamental differences in structure and in cortical representation. Language is constructed of subunits which are organised in phonological and grammatical structure. This is true for both words and signs, despite their very different surface appearance. Signs have a phonology in which elements such as hand shape and location contrast with each other in the same way as the phonemes of spoken language. Although very similar in appearance to signs, gestures are holistic, semantic expressions without comparable substructure. The absence of grammatical structure can be seen in the very example of instrumental gestures (Armstrong et al. 1995) referred to by Corballis, which cannot be differentiated into noun, verb, or sentence.
Gestures are much less strongly lateralised, and the cortical areas involved do not overlap closely with the areas involved in language, whether spoken or signed. Although gesture and sign language use the same modality, observation of communication abilities following brain injury exhibits a clear dissociation (Corina et al. 1992; Hickok et al. 2002, Marshall et al., in press). Left-hemisphere injury strongly impairs signing, regardless of the degree of sign iconicity, whereas gestures remain largely intact. In contrast, right-hemisphere injury leaves most features of sign language intact, even in the presence of substantial visuospatial impairment (Atkinson et al., in press; Corina et al. 1999; Loew et al. 1997). This dissociation also highlights the different processing capacities of the left and right hemispheres (in adults).

We suggest that this evidence speaks against the occurrence of a gestural protolanguage; although speech and language developed from vocalisations that accompanied gesture, gesture itself did not achieve linguistic structure. There are also several lines of evidence to suggest that sign language was not an intermediate step between gesture and speech, the most striking being that both signed and spoken language are processed in the same regions of the left auditory cortex (MacSweeney et al. 2001; 2002). The later-evolved communication was built on cortical areas used in earlier forms of communication. In analogy to the communicative twinning of gesture and speech, sign language may be accompanied by distinct syllabic vocal gestures (echo phonology) (Woll 2001).

We agree with Corballis that there must be an evolutionary reason why both language and hand dominance are predominantly located in the left hemisphere, but we caution against the comparison with anatomical lateralisation. Although there may be subtle differences in the volume of specific homotopic areas in the mature human brain, it is well known that the right hemisphere can assume the functions of the dominant left side following injury in early childhood. Both halves of the brain are pluripotential; the observed differences are not organic but functional-developmental, possibly related to different maturational rates of the hemispheres. Although left-hemisphere dominance for specific functions was established early in evolution, this has occurred without leaving any convincing anatomic trace.

We consider that hemispheric lateralisation is related to a fundamental neurobehavioural division that occurred early in evolution. The left hemisphere has become specialised for targetdirected behaviour (including vocalisation), whereas the right hemisphere is specialised for monitoring of the environment (Sieratzki \& Woll 2002).

As a result, the left hemisphere directs sequential processing, and the right hemisphere controls holistic spatial processing. It is therefore reasonable to hypothesise that target-directed vocalisations became localised in the left hemisphere and that later, the particular capacity of Broca's area to map perception onto execution, to which Corballis refers, led to its becoming the site of language. Handedness is less strongly determined by an overall neurobehavioural disposition, with a variety of independent determinants (genetic, ergonomic, social) coming into play.

In conclusion, we suggest that the contrasting functional specialisation of the hemispheres reflects the fundamental duality of behavioural challenges that a species faces in its interaction with the environment. The homolaterality of language and hand dominance is more an outcome of this specialisation rather than the expression of a specific evolutionary link. 